\title{
BUILDING A 3D REFERENCE MODEL FOR CANAL TUNNEL SURVEYING USING SONAR AND LASER SCANNING
}

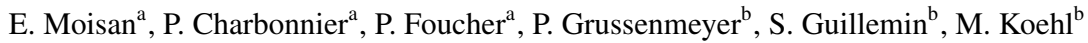 \\ ${ }^{a}$ Cerema Dter Est, ERA27 IFSTTAR, 11 rue Jean Mentelin, B.P. 9, 67035 Strasbourg, France \\ (Emmanuel.Moisan, Pierre.Charbonnier, Philippe.Foucher)@ cerema.fr \\ ${ }^{\mathrm{b}}$ ICube Laboratory UMR 7357, Photogrammetry and Geomatics Group, INSA Strasbourg, 24 Boulevard de la Victoire, \\ 67084 Strasbourg, France - (pierre.grussenmeyer, samuel.guillemin, mathieu.koehl)@ insa-strasbourg.fr
}

\section{Commission V}

KEY WORDS: 3D Bathymetry, Data processing and 3D Modeling, Reference control and accuracy assessment

\begin{abstract}
:
Maintaining canal tunnels is not only a matter of cultural and historical preservation, but also a commercial necessity and a security issue. This contribution adresses the problem of building a full 3D reference model of a canal tunnel by merging SONAR (for underwater data recording) and LASER data (for the above-water parts). Although both scanning devices produce point clouds, their properties are rather different. In particular, SONAR data are very noisy and their processing raises several issues related to the device capacities, the acquisition setup and the tubular shape of the tunnel. The proposed methodology relies on a denoising step by meshing, followed by the registration of SONAR data with the geo-referenced LASER data. Since there is no overlap between point clouds, a 3-step procedure is proposed to robustly estimate the registration parameters. In this paper, we report a first experimental survey, which concerned the entrance of a canal tunnel. The obtained results are promising and the analysis of the method raises several improvement directions that will help obtaining more accurate models, in a more automated fashion, in the limits of the involved technology.
\end{abstract}

\section{INTRODUCTION}

In France, there are 33 canal tunnels still in service for commercial navigation and boating, representing $42 \mathrm{~km}$ of underground waterway. The preservation of those structures, mostly bored during the $19^{\text {th }}$ and $20^{t h}$ centuries, aims at conserving a heritage and at protecting goods and persons. Hence, periodical inspections are necessary. However, on-site visual investigations are time-consuming and difficult to put into practice. In this context, Voies Navigables de France (VNF, the French operator of waterways), the Centre d'Étude des tunnels (CETU) and the Cerema, in collaboration with the Photogrammetry and Geomatics Group (INSA) are devising an automatic inspection system based on image and SONAR recording. A photogrammetric acquisition prototype was initially developed for dynamically surveying the above-water part of tunnels (Charbonnier et al., 2014), see Fig. 1. In the future, an underwater recording device will complement the system. To assess the accuracy of such a mobile recording system, it is necessary to build a reference model of the whole tunnel (i.e. including its under and above-water parts).

For the purpose of building an accurate reference model, we proceed to static acquisitions along the tunnel. For the above-water parts, a 3D Terrestrial LASER Scanner (TLS) was selected for its ability to get accurate and easily geo-referenced data (Charbonnier et al., 2014). For the underwater part, a 3D Mechanical Scanning SONAR (MSS) was chosen because the turbidity of the canal water excludes optical solutions. It enables scanning the environment from stations in the same manner as a TLS, see e.g. (Drap et al., 2011, Sohnlein et al., 2011).

Both devices automatically provide point clouds through a mechanical scanning system. However, while processing and georeferencing TLS data to obtain a 3D model is rather straightforward, handling MSS data is more complicated. In particular, MSS data are very noisy, which requires robust reconstruction and registration methods to obtain a 3D model of the underwater

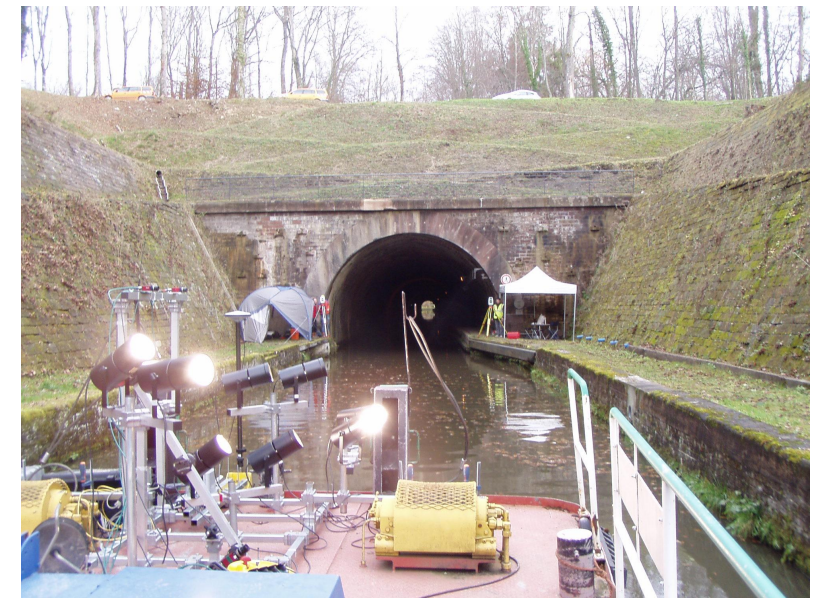

Figure 1: Modular on-board mobile image recording system on the experimental site.

part of the structure. Moreover, in practice, the absolute location and orientation of the SONAR is not available, so a direct geo-referencing of MSS data is impossible. Hence, we proceed in an indirect manner, by co-registering the above and underwater models, which provides a geo-referenced full 3D model of the canal tunnel.

Another difficulty arises from the fact that the above and underwater point clouds do not overlap. This makes the co-registration task more complex. Fortunately, we can exploit some geometric primitives (planes, lines) which are common to its above and underwater parts of the structure. Moreover, we immersed two wooden ladders, that we robustly fitted in both models to provide additional constraints to the registration algorithm.

The rest of the paper is organized as follows. We first propose a 
brief overview of related works in Sect. 2. Then, in Sect. 3, we show how we use TLS and MSS for data recording. In Sect. 4, we focus on the meshing of MSS data to filter noise and obtain a 3D model of the underwater part of the tunnel. Sect. 5 is dedicated to the construction of the full 3D model by co-registering the above and underwater models. In Sect. 6, we comment the experimental results and propose future directions for this work.

\section{RELATED WORK}

Partly immersed structures are exposed to water conditions in addition to classical degradations. Therefore, surveying systems were recently developed to detect anomalies, both under and above the surface. In some works, typically for dams or harbors inspection, LASER and SONAR acquisitions are combined. Such techniques are based on dynamic acquisitions and involve Inertial Navigation Systems and Global Positioning Systems (INS+GPS) to monitor the attitude and localization parameters of the platform. This approach is for instance used in (Mitchell et al., 2011) and (Rondeau et al., 2012). Of course, GPS signals are not available in tunnels, so other solutions must be sought. In (Papadopoulos et al., 2014), the authors propose a Simultaneous Localisation And Mapping (SLAM) technique that relies on the registration of TLS point clouds, thanks to the Iterative Closest Point (ICP) algorithm. However, the tubular shape of the tunnel is a disadvantage for this method.

In the previously mentioned works, both TLS and MSS sensors are embedded on a common, mobile platform, which is not the case in our application: we are faced with the problem of locating individual MSS acquisitions. Such problem is similar to Remotely Operated Vehicle (ROV) and Autonomous Underwater Vehicule (AUV) localisation. In (Ridao et al., 2010) a triangulation system based on acoustic is proposed. The apparatus is composed of surface buoys equipped with ultra-short baseline transducers (USBL) tied up with GPS receivers. Another frequently employed method is visual odometry (Scaramuzza and Fraundorfer, 2011, Scaramuzza and Fraundorfer, 2012), from optical or acoustic imaging in addition to SLAM algorithms see (Guth et al., 2014) and references therein, but it is not suited to static acquisitions. A simpler problem is the relative positioning of MSS data, for which the more commonly used algorithm is ICP (Dobke et al., 2013, Drap et al., 2011) or its robust version.

\section{DATA RECORDING}

The experimental acquisitions have been carried out in a canal tunnel located in Niderviller, Lorraine region, France. The tunnel (see Fig. 1), was constructed between 1839 and 1845. It is straight and lined with stonework. It is $475 \mathrm{~m}$ long and, for practical reasons, the acquisitions were focused on its entrances, in this first experimental campaign.

\subsection{Laser scanning}

The above-water acquisitions were performed using a Faro ${ }^{\circledR}$ Focus 3D TLS. To survey the environment in 3D, a laser beam sweeps the visible surface, vertically and horizontally. Several solutions are possible to get the distance between the TLS and the object. The one implement in the Focus involves measuring the phase difference between the emitted and received pulse.

For each entrance, two stations have been performed, from each bank of the canal, the same day as the underwater acquisition. The model can be complemented using a previously performed

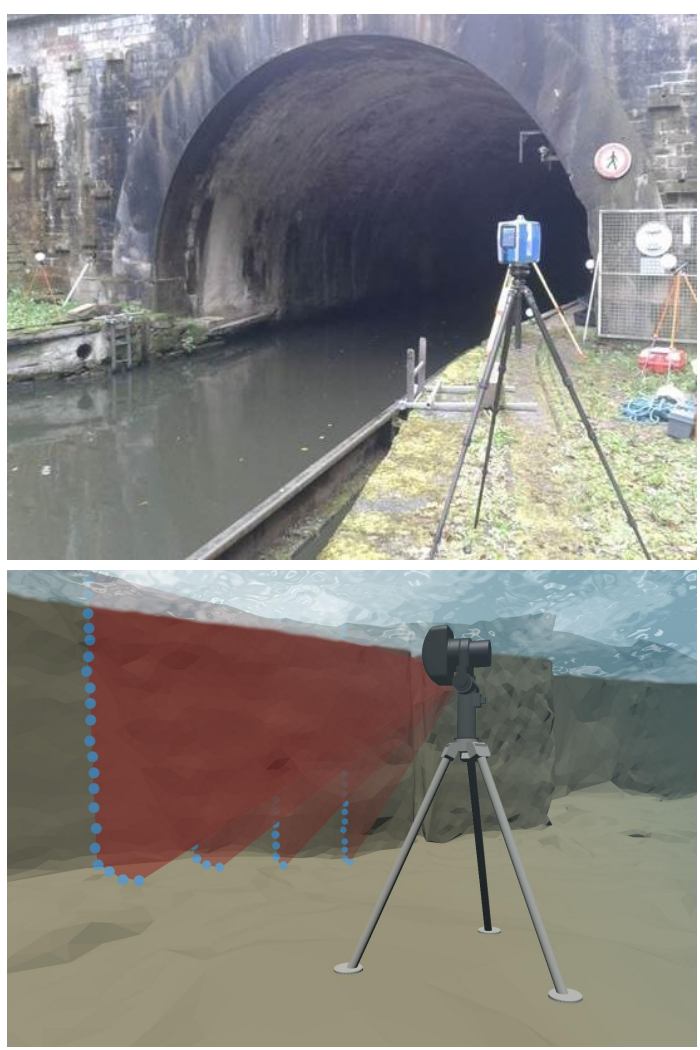

Figure 2: Data acquisitions in Niderviller's tunnel : photograph of TLS device and spherical targets (top); schematic representation of MSS devices (bottom)

TLS surveying of the whole tunnel. To register point clouds, spherical targets have been placed in the shared scanning area, as shown in Fig. 2 (top). In order to geo-reference the model, the coordinates of sphere centers have been determined with traditional surveying methods based on a set of points known in the French reference coordinate system (RGF 93 and NGF-IGN 69) implemented on the site.

\subsection{SONAR scanning}

The underwater acquisition was carried out with the Blueview ${ }^{\circledR}$ BV5000 MSS, see Fig 2 (bottom). The device is made up of a multi-beam echo-sounder with vertical swath direction, and of a rotation system with vertical axis, enabling a $360^{\circ}$ horizontal scan. Since the swath aperture is $45^{\circ}$, a mechanical system is used to tilt the sensing head so potentially a $320^{\circ}$ vertical range can be scanned. The acoustic sensor emits high frequency signal (1.35 Mhz) which offers a good distance resolution but at the same time limits the maximum acquisition range to $30 \mathrm{~m}$. According to the constructor's data sheet, the vertical and horizontal resolution at $10 \mathrm{~m}$ are $16 \mathrm{~mm}$ and $30 \mathrm{~mm}$, respectively.

Two stations placed at $10 \mathrm{~m}$ from each other have been performed on each entrance of the tunnel, one inside and one outside it, see Fig. 3. Two wooden ladders ( $3.60 \mathrm{~m}$ high $0.32 \mathrm{~m}$ wide) and were partly immersed in the water, so they were recorded by both the MSS and the TLS. This will enable the registration of the underwater and above-water models.

\subsection{Comparison of TLS and MSS devices}

The comparison of device features (see Tab. 1) highlights the differences that the recorded point clouds show. We can note a 


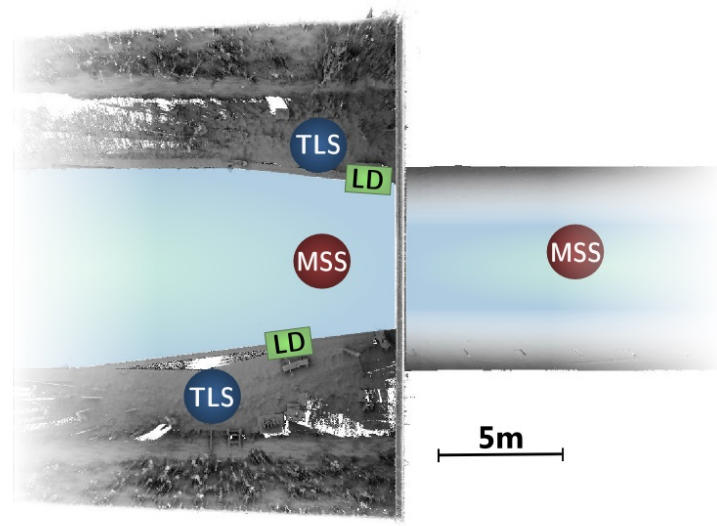

Figure 3: Illustration of the acquisition setup seen from above, featuring TLS and MSS stations, as well as the location of ladders (LD)

large difference in spatial resolution, i.e. the distance between a recorded point and its closest neighbor. But the main contrast concerns the beam width which has a direct impact on the imagery resolution, i.e. the ability to distinguish two echoes coming from two different targets. For exemple, the signal footprint on a plane perpendicular to the signal direction $10 \mathrm{~m}$ away from the device is a circle of $6 \mathrm{~mm}$ diameter for the Focus 3D and a square of $175 \mathrm{~mm}$ side length for the BV5000. In addition, the more the incident angle of the beam, the more these dimensions increase. In the case of canal tunnels, which are elongated structures, the incidence angle quickly becomes unfavorable. This influence is visible on the acoustic images, shown on Fig. 4. One can see that the vertical line, which corresponds to the footprint of the swath on the canal's wall is much wider for a small grazing angle of acquisition than for an almost perpendicular acquisition. According to a theoretical model of the acquisition setup and to the datasheet of the BV5000, we estimate horizontal width of the beam footprint (see Fig. 5). We see that, in the case of canal tunnels, this length can easily reach more than $0.5 \mathrm{~m}$.
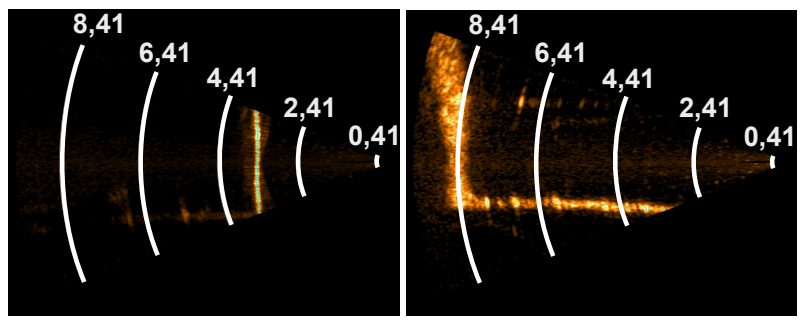

Figure 4: Multi-beam echo-sounder swaths under two different incidence angles. Left: almost perpendicular incidence (see red line in Fig. 5); Right: small grazing angle (see green line in Fig. 5)). The swath is wider in the second case. Distances are given in meters

Another important difference between terrestrial and underwater imaging is that in the latter case, no visual control can be made in practice. Hence, if the MSS moves during the acquisition, there is no way of knowing it. This might lead to artifacts or inconsistent measurements.

\section{3D SURFACE RECONSTRUCTION}

Most of the time in surveying applications, surface reconstruction is performed by post-processing to obtain a simplified dig-
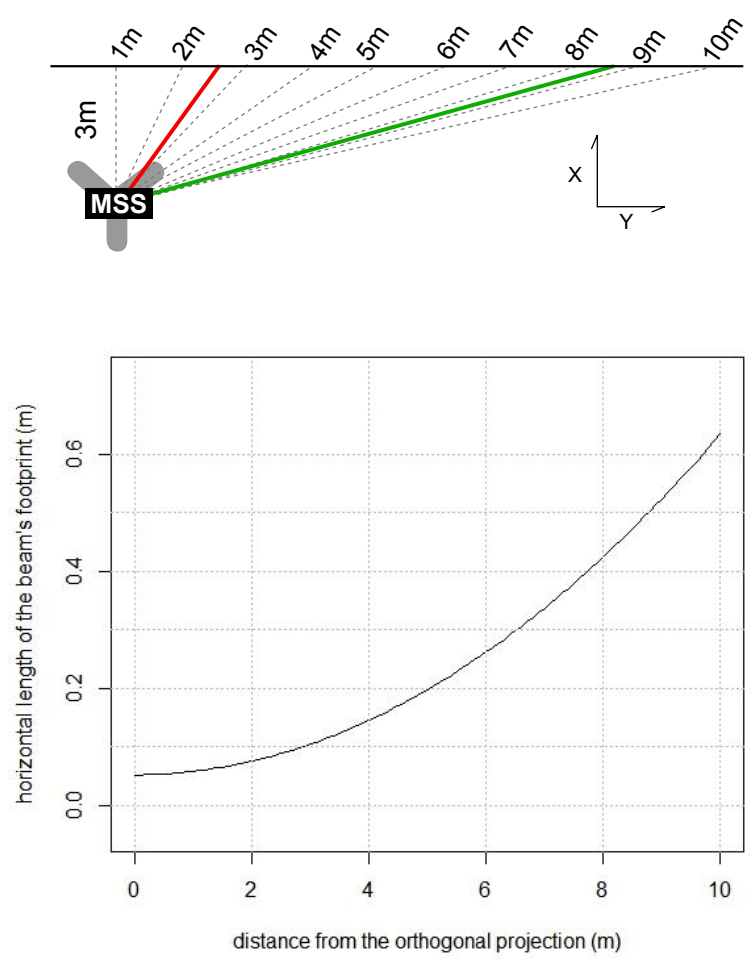

Figure 5: Top-view of the theoretical acquisition setup (top); Estimation of the SONAR horizontal resolution as a function the distance from the orthogonal projection (bottom)

ital model of structures. The surface can be reconstructed from the point cloud, by detecting geometric primitives or by meshing, which is better suited to complex geometries. In our case, we are faced with very noisy data. Hence, we use meshing as a preprocessing, mainly for denoising the underwater model. There are two main ways of processing surface reconstruction by meshing. The first one uses cloud points as vertices for triangulation. Such method is obviously sensitive to noise, so it cannot be directly applied to MSS data. A first solution to this problem is to filter the point cloud before meshing, for example by selecting evenly spaced points. A second one is to compute the nearest surface to points using robust estimators, at the risk of obtaining an over-smooth model. In fact, with both cases, there is a risk of loosing details. The choice of the method heavily relies on the properties of the point cloud. As noticed in the previous part, the above and underwater recorded data present many character-

Table 1: TLS and MSS acquisition parameters

\begin{tabular}{|l|l|l|}
\hline & $\begin{array}{l}\text { Faro Focus 3D } \\
\text { X330 }\end{array}$ & Blue View BV5000 \\
\hline $\begin{array}{l}\text { horizontal reso- } \\
\text { lution }\end{array}$ & $0.036^{\circ}$ & $\begin{array}{l}\sim 0.09^{\circ} \\
(16 \mathrm{~mm} / 10 \mathrm{~m})\end{array}$ \\
\hline $\begin{array}{l}\text { vertical resolu- } \\
\text { tion }\end{array}$ & $0.036^{\circ}$ & $\begin{array}{l}0.18^{\circ} \\
(30 \mathrm{~mm} / 10 \mathrm{~m})\end{array}$ \\
\hline beam width & $\begin{array}{l}2.25 \mathrm{~mm}+2 \times \\
0.011^{\circ}\end{array}$ & $1^{\circ} / 1^{\circ}$ \\
\hline ranging error & $2 \mathrm{~mm}(10-25 \mathrm{~m})$ & $15 \mathrm{~mm}$ \\
\hline $\begin{array}{l}\text { maximum } \\
\text { range }\end{array}$ & $330 \mathrm{~m}$ & $30 \mathrm{~m}$ \\
\hline field-of-view & $300^{\circ} / 360^{\circ}$ & $45^{\circ} / 360^{\circ}$ \\
& & $\left.320^{\circ} / 360^{\circ}\right)$ \\
\hline
\end{tabular}


istic differences, so the suitable reconstruction methods are also different.

The meshing of both the MSS and TLS data has been made using 3DReshaper ${ }^{\circledR}$. Both previously mentioned meshing techniques are implemented in the software. The advantage of this program is to allow surface reconstruction by successive refinements using the point cloud.

As previously mentioned, MSS data are very noisy, so the underwater model reconstruction was performed in a coarse-to-fine manner. A first large-scale mesh is made by selecting points in the mesh according to a distance criterion. Then, the resolution of the mesh is progressively increased by picking points again in the cloud or by interpolating new points. Point selection involves either a distance-to-mesh or a maximum surface deviation criterion. These parameters must be carefully chosen. In this first experiment, they are manually tuned by an operator.

Unlike MSS point clouds, TLS point clouds have a negligible noise for points located at a certain distance from the device as well as for certain target material properties. Thus, to use maximum of point cloud informations, all points (except outliers) have been employed as triangle vertices to reconstruct the surface.

We note that this step of the process requires many manual operations like outlier removal or the correction of mesh reconstruction mistakes. While such interventions can be supported by photographs or other physical measurements for TLS data, this is of course not the case for underwater data. Hence, the construction of the underwater model from MSS data involves an important part of interpretation.

\section{REGISTRATION AND GEO-REFERENCING}

Both the TLS and the MSS provide point clouds that must be registered in order to obtain a geo-referenced 3D model.

In general, there are two ways of registering and geo-referencing point clouds. The first one is direct: it requires the knowledge of the position of the scanner. This can be obtained a priori, by placing the device at a point of known coordinates, or a posteriori, by surveying its position using conventional techniques. The second one is indirect: the point clouds are first co-registered and then, the geo-referencing is made using elements of the cloud with known coordinates. The registration of point clouds can be based either on targets or on clouds.

Target-based registration requires to anticipate and place targets in the field of view. Their geometry and scale depend on the spatial resolution and precision of the scanner. Spheres are usually used for TLS recording because the determination of their centers can be made very accurately. Of course, the quality of the registration also depends on the distribution and number of targets. This method is very usual in lasergrammetry and is the one that we chose for our application.

Cloud-based registration involves to automatically find homologous points between scans, and then, to compute the geometric transformation (rotations, translations) between sets of points. The most popular algorithm is the ICP (Iterative Closest Point) method, introduced by (Besl and McKay, 1992), which iteratively minimizes the difference between the first set of points and the geometric transformation of the second set of points. This method requires a certain overlap between point clouds and also, a first estimation of the transformation. An alternative technique is based on finding homologous geometrical entities between scans. These entities can be planes, spheres, cylinders or lines and to find the best way to align them.

\subsection{Underwater registration}

Registration and geo-referencing are more complicated for the BV5000 scans than for TLS data. Since, in our setup, the position and orientation of the underwater scanner cannot be directly determined, we have to resort to the indirect method. Cloud-tocloud registration seems to be the easiest technique to implement but it also raises several issues. Some are due to the nature of the technology itself: MSS data are very noisy and the resolution is rather coarse, so finding correspondences is difficult. A second issue is related to the elongated shape of the canal and to our experimental setup, that produce small grazing angles: the farther the point, the less the accuracy, which also influences the quality of registration. In these conditions, it is very difficult to resolve the longitudinal ambiguity, i.e. to accurately estimate the translation along the tunnel axis. Immersing geometric reference objects (e.g. ladders) or decreasing the distance between stations to increase the overlap quality are possible solutions to alleviate this problem. In our case, we found it more practical to register MSS data with TLS data as explained in more details in the above sub-section.

\subsection{Underwater geo-referencing}

As the geo-referencing of TLS data is straightforward, we chose to register MSS point clouds on TLS acquisitions to get a georeferenced global model. The main issue is the lack of overlap between TLS and MSS acquisition, which prevents from using cloud-to-cloud registration directly, hence we split up the problem into three steps that are described below.

5.2.1. Attitude correction There is no common scan zone between under and above water acquisitions, but certain parts of both models can be approximated by a same geometrical entity. For example, planes can approximate some parts of canal banks and projecting elements can be defined by lines. We use plane normals and line directions to estimate the rotation matrix. A minimum of two non-collinear vectors are required to get the attitude corrections.

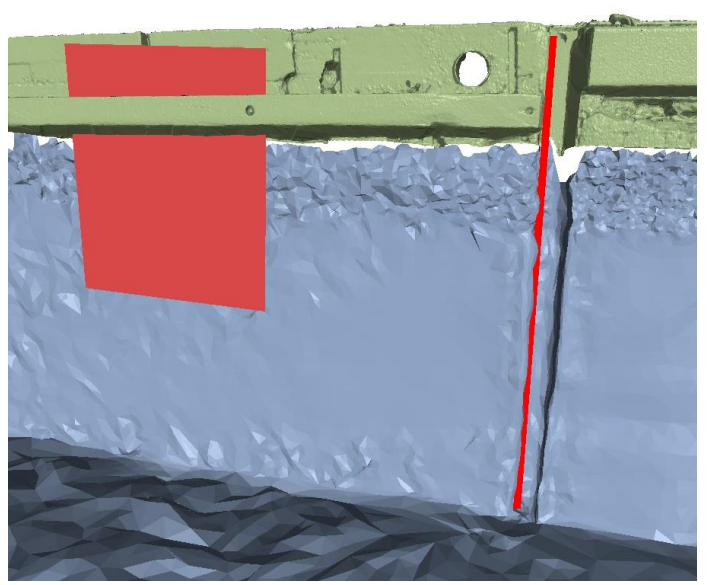

Figure 6: Geometric entities (plane, line) for attitude correction

A property of the rotation matrix that must be taken into account in the computation is its orthogonality. We use the solution described in (Golub and Van Loan, 2012) who suggest an answer to the so-called "orthogonal Procrustes problem":

$$
\min \|A-B Q\|_{F}^{2} \quad \text { subject to } Q^{T} Q=I
$$


were $\|.\|_{F}$ denotes the Frobenius norm, and where A and B are respectively the direction vectors of the elements extracted from the TLS and the MSS models and Q is the $3 \times 3$ rotation matrix. The algorithm computes the Singular Value Decomposition (SVD) of the $B^{T} A$ product, $U^{T}\left(B^{T} A\right) V=\Sigma$, to get the rotation matrix $Q$, as:

$$
Q=U V^{T}
$$

5.2.2. Vertical translation During this step, we compute the vertical translation using the ladders, whose dimensions are known. More specifically, we use the positions of the ladder rungs in both models to estimate the difference in altitude. But the underwater acquisition provides noisy point clouds, so the information cannot be directly extracted from the point cloud. Hence, to get an accurate result we developed a robust estimation method to model the ladder.

The first step of the method is the segmentation of the underwater acquisition to extract the ladder points from the MSS point cloud. Then, the ladder plane is computed using a robust version of Principal Component Analysis, based on a M-estimator. Finally the points of the ladder cloud are projected onto the plane, so the rest of the process is performed in two dimensions.

The second step is the automatic segmentation of ladder's components (vertical bars and rungs) thanks to histogram analysis. The previous step provides a $2 \mathrm{D}$ point cloud in a coordinate system with abscissa axis parallel to the vertical bars and ordinate axis parallel to the rungs. The statistical distributions of point coordinates along both axes show peaks that allow an easy segmentation of the cloud. Hence, points are split into clusters, that correspond either to vertical bars or to rungs.

Then, the third step is the robust adjustment of straight lines on the data, to model the bars and rungs. For this purpose, we use a linear regression and we simultaneously fit all the lines, taking into account that rungs are parallel, that the two vertical bars are also parallel, and that vertical bars are perpendicular to rungs. Such constraints are very useful in our context, where data are very noisy. In this experiment, we use an orthogonal fit, i.e. the residual is the orthogonal distance between the point and the model. One may note that affine fitting could also be used because the components of the ladder are almost parallel to axes. In both cases, we use a M-estimator, i.e. we replace the usual sum of squared residuals by a function of the form:

$$
J(\theta)=\sum_{i} \rho\left(r_{i}\right)
$$

where $\theta$ is the vector of model parameters, $\rho$ is a non-quadratic potential or penalty function and $r_{i}$ is the residual, i.e. the difference between the observation and its prediction according to the model. In the half-quadratic setup, see e.g. (Charbonnier et al., 1997), it is shown that minimizing $J$ is equivalent to minimizing

$$
J^{\star}(\theta, b)=\sum_{i} b_{i} r_{i}^{2}+\Psi\left(b_{i}\right)
$$

where $\Psi$ is a convex the cost function whose expression can be related to $\rho$ and $b_{i}$ is an auxiliary variable, whose role is both to mark outliers and to linearize the problem. Indeed, $J^{\star}$ is quadratic with respect to $r$ (hence, w.r.t. $\theta$ in linear regression) when $b$ is fixed, and convex w.r.t. $b$ when $r$ is fixed. Moreover, in the latter case, the minimum is obtained for $b=\rho^{\prime}(r) / 2 r$. Such properties suggest a deterministic algorithmic strategy that consists in alternately fixing each variable and minimizing w.r.t. the other.
In the affine regression setup, this leads to the well-known Iterated Reweighted Least-squares (IRLS) algorithm. This strategy may be extended to the simultaneous fit of several curves, see e.g. (Tarel et al., 2007, Tarel et al., 2008). However, the algorithm we obtain here is slightly different because of the additional parallelism and orthogonality constraints related to the geometry of ladders. In the orthogonal setup, the resulting algorithm simultaneously estimates the centroids of bars and rungs in an IRLS fashion, and their direction using a reweighted version of PCA.

Finally, the intersection points between rungs and vertical bars are calculated and transformed back in 3D coordinates.

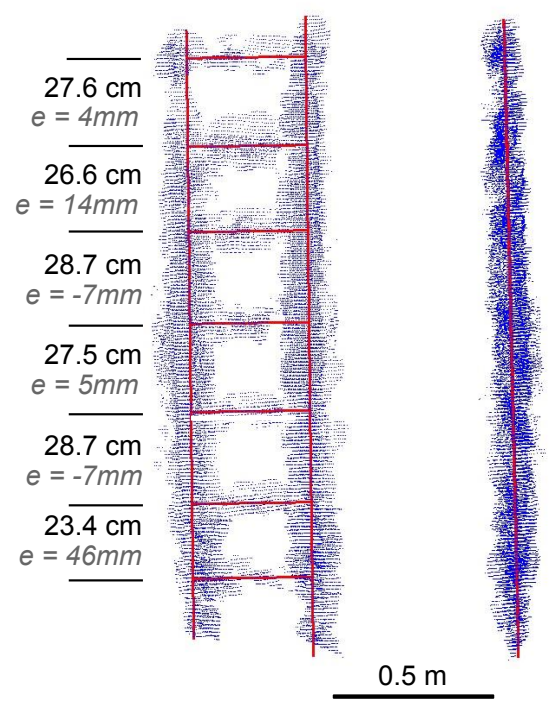

Figure 7: Ladder fitting. Left: front view with inter-rung distances and errors (e) with respect to ground truth. Right: sectional view showing the adjustment of the ladder plane.

Fig. 7 shows an example of robust ladder fitting. One may see that, despite the strong noise level, the ladder is well approximated (the maximum error with respect to ground truth measurements of inter-rung distances is $46 \mathrm{~mm}$ and most errors are less than $10 \mathrm{~mm}$ ).

5.2.3. Horizontal translation Once the attitude and vertical translation have been correctly estimated, the last operation consists in estimating the horizontal translation vector. The $2 \mathrm{D}$ silhouette of the waterline along the structure can be extracted on both the TLS ans MSS model by intersecting the corresponding point clouds with the plane that corresponds to the water surface. Finally, we apply a 2D ICP algorithm to estimate the translation vector between the two models.

5.2.4. Final result The estimated transformation can be applied to the underwater meshed model as well as to the original point cloud. Indeed, the underwater point cloud provides visual information that may disappear in the mesh view. For example, projected shadow highlights certain elements of the scene. Fig. 8 shows the result that we obtain. Overall, the obtained model is visually satisfying, even if some imperfections may be observed in regions far from the station. One may see e.g. at the bottom left of the topmost image, that the underwater model has a very granular aspect and that the adjustment of both models becomes less perfect. These defects are probably due to the fact that the footprint of the beam is very large at such a distance from the source. 

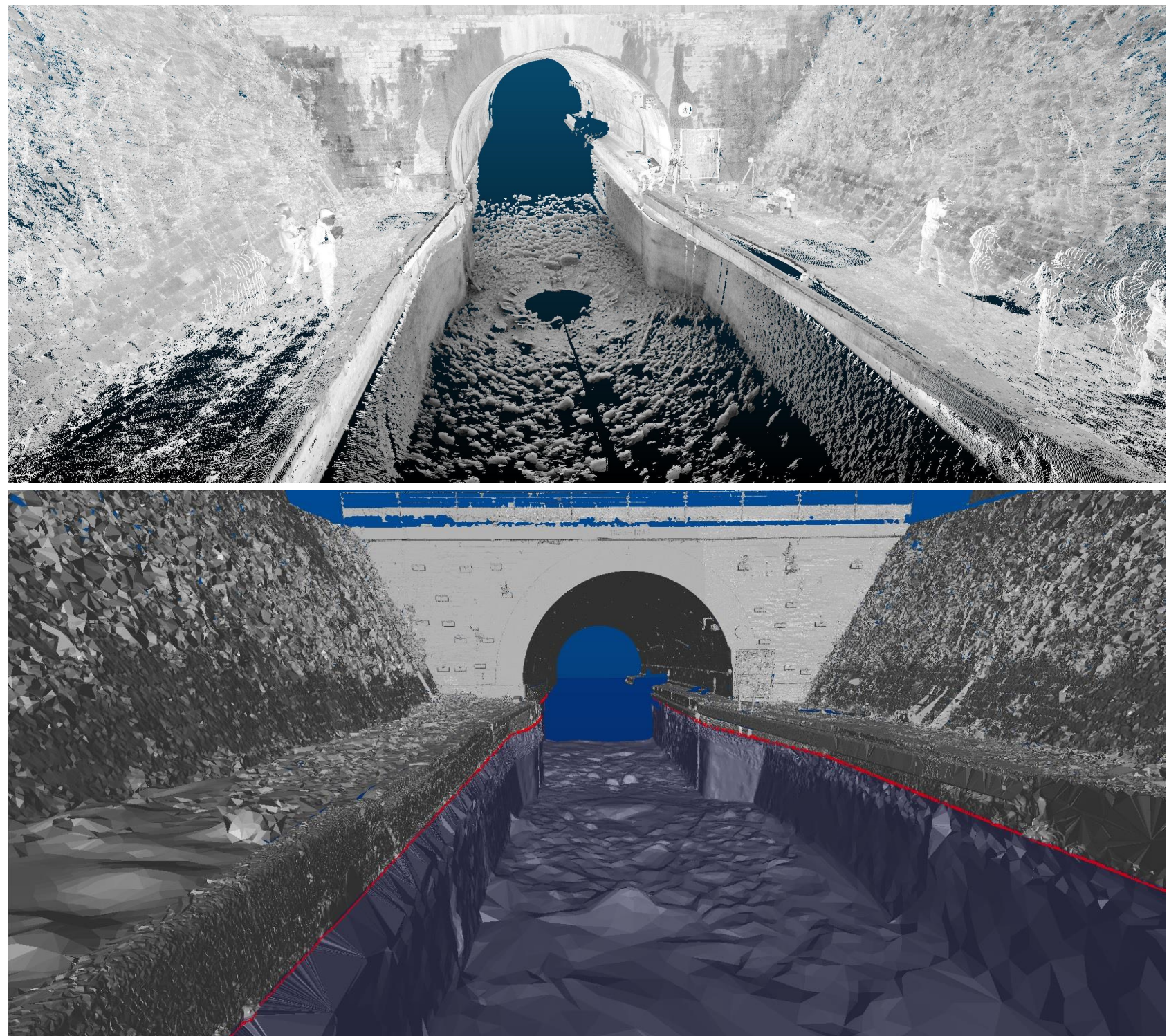

Figure 8: Resulting full 3D geo-referenced model of Niderviller's canal tunnel entrance. Top: point cloud visualization; bottom: mesh visualisation (the blue mesh is the underwater model and the red line defines the waterline).

\section{DISCUSSION}

In this paper, we have introduced a method to record and process TLS and MSS data with the purpose of building a 3D reference model of a canal tunnel. A comparison between the SONAR and LASER device characteristics highlights differences that impact the quality of the data. In particular, it appears that MSS data have a much lower resolution than TLS data, and that the angular loss of resolution can be rather strong. Since the elongated shape of the tunnel generates unfavorable incidence angles, the quality of SONAR acquisitions becomes rather coarse at large distances from the scanner. Moreover, this technology tends to provide noisy data.

To limit the effect of perturbations on the processing, we first perform a data denoising by meshing. More specifically, a coarseto-fine method, which gradually increases the resolution of the mesh, has been used. Of course, this step removes noise, but at the same time some details can be lost. In this experiment, the trade-off between noise and details was made empirically, which is difficult. Contrary to TLS data, there is no direct visual or physical reference that could guide the process: MSS acquisitions are actually the only visual information available. It might be envisioned to infer the underwater texture by assuming that there is a continuity with the above-water part, but this is not satisfactory. We believe that acoustic and image processing techniques should be explored to devise a more automatic and data-driven denoising technique. In particular, moving least squares, bilateral filtering (Tomasi and Manduchi, 1998), non-local means filtering (Buades et al., 2005, Deschaud and Goulette, 2010, Digne, 2012) or structure+texture decompositions (Aujol et al., 2006) seem appealing for this task.

A second important issue is related to the co-registration of the point clouds provided by the scanners. While processing TLS data to obtain the above-water geo-reference 3D model is rather straightforward, this task is much more difficult for MSS data. Due to the low resolution of the data and to the angular loss of resolution, classical techniques such as ICP did not provide satisfactory results in our experiments. To alleviate this problem, its is possible to improve the experimental setup by reducing the distance between MMS stations: an interval of about $5 \mathrm{~m}$ would be recommended. Moreover, it is possible to immerse more tar- 
gets, such as the ladders that we used here, to provide additional references to help the registration. Our experiments show that the targets must be carefully chosen and placed on-site: for example, the ladders should be separated from the canal walls, unless their segmentation becomes problematic.

To obtain a geo-referenced global model of the canal-tunnel, registering MSS and TSS data is a crucial point. The main difficulty we are faced with in this application is that there is no overlap between the above and underwater models. We therefore proposed a 3-step procedure in which, first, geometrical entities are used to fix the attitude parameters. The second step uses the ladders to estimate the vertical correction: we proposed a robust method based on M-estimation to fit lines on the bars and rungs of the ladder using the noisy point cloud. We assessed the method by comparing the results of the fit with direct measurement of distances. In most cases, the error is less than $10 \mathrm{~mm}$ (with a maximum of $46 \mathrm{~mm}$ ). Third, after having extracted the intersection lines between the water surface and the MSS and TLS models, we apply a 2D ICP algorithm limited to estimate the remaining 2D translations.

This experimentation provides an initial overview of underwater acquisition in canal tunnel and yields promising results. Improvements of the model's quality may be expected from a better experimental setup (closer stations, more numerous targets). More automatic, data-driven filtering technique should help enhancing the quality of the data and limit manual interventions. Many problems we are faced with result from intrinsic limitations of the acquisition techniques, but one may foresee that the technology will progress, so we may expect increasingly accurate results using the proposed methodology. The obtained models will be a reference for future acquisitions, in static (to get the tunnel deformation) or with dynamic underwater acquisitions systems (for assessing mobile mapping solutions).

\section{REFERENCES}

Aujol, J.-F., Gilboa, G., Chan, T. and Osher, S., 2006. Structuretexture image decomposition-modeling, algorithms, and parameter selection. International Journal of Computer Vision 67(1), pp. 111-136.

Besl, P. and McKay, N. D., 1992. A method for registration of 3-D shapes. Pattern Analysis and Machine Intelligence, IEEE Transactions on 14(2), pp. 239-256.

Buades, A., Coll, B. and Morel, J.-M., 2005. A non-local algorithm for image denoising. In: Proceedings of the 2005 IEEE Computer Society Conference on Computer Vision and Pattern Recognition (CVPR'05) - Volume 02, CVPR '05, pp. 60-65.

Charbonnier, P., Blanc-Féraud, L., Aubert, G. and Barlaud, M., 1997. Deterministic edge-preserving regularization in computed imaging. IEEE Transactions on Image Processing 6(2), pp. 298311 .

Charbonnier, P., Foucher, P., Chavant, P., Muzet, V., Prybyla, D., Perrin, T., Albert, J., Grussenmeyer, P., Guillemin, S. and Koehl, M., 2014. An image-based inspection system for canal-tunnel heritage. International Journal of Heritage in the Digital Era 3(1), pp. 197-214.

Deschaud, J.-E. and Goulette, F., 2010. Point cloud non local denoising using local surface descriptor similarity. In: N. Paparoditis, M. Pierrot-Deseilligny, C. Mallet and O. Tournaire (eds), IAPRS, Vol. XXXVIII, Part 3A, Saint Mand, France, pp. 109114.
Digne, J., 2012. Similarity based filtering of point clouds. In: Computer Vision and Pattern Recognition Workshops (CVPRW), 2012 IEEE Computer Society Conference on, pp. 73-79.

Dobke, A., Vasquez, J., Lieu, L., Chasnov, B., Clark, C., Dunn, I., Wood, Z. J. and Gambin, T., 2013. Towards threedimensional underwater mapping without odometry. In: Proceedings of Unmanned Untethered Submersible Technology Conference, Portsmouth, New Hampshire, USA.

Drap, P., Merad, D., Boï, J.-M., Boubguira, W., Mahiddine, A., Chemisky, B., Seguin, E., Alcala, F. and Bianchimani, O., 2011. ROV-3D: 3D underwater survey combining optical and acoustic sensor. In: Proceedings of the 12th International Conference on Virtual Reality, Archaeology and Cultural Heritage, VAST'11, Eurographics Association, Aire-la-Ville, Switzerland, pp. 177184.

Golub, G. H. and Van Loan, C. F., 2012. Matrix computations. Johns Hopkins Studies in the Mathematical Sciences, Vol. 3, Johns Hopkins University Press; $4^{\text {th }}$ edition.

Guth, F., Silevira, L., Botelho, S., Drews, P. and Ballester, P., 2014. underwater SLAM: Challenges, state of the art, algorithms and a new biologically-inspired approach. In: 5th IEEE RAS \& EMBS International Conference on Biomedical Robotics and Biomechatronics (BioRob), São Paulo, Brasil, pp. 981-986.

Mitchell, T., Miller, C. and Lee, T., 2011. Multibeam surveys extended above the waterline. In: Proceedings of WEDA technical conference and Texas A\&M dredging seminar, Nashville, Tennessee, USA, pp. 414-422.

Papadopoulos, G., Kurniawati, H., Shariff, A. S. B. M., Wong, L. J. and Patrikalakis, N. M., 2014. Experiments on surface reconstruction for partially submerged marine structures. Journal of Field Robotics 31(2), pp. 225-244.

Ridao, P., Carreras, M., Ribas, D. and Garcia, R., 2010. Visual inspection of hydroelectric dams using an autonomous underwater vehicle. Journal of Field Robotics 27(6), pp. 759-778.

Rondeau, M., Leblanc, E. and Garant, L., 2012. Dam infrastructure first inspection supported by an integrated multibeam echosounder MBES)/LiDAR system. In: Proceedings of the Canadian Dam Association annual conference, Saskatoon, Canada.

Scaramuzza, D. and Fraundorfer, F., 2011. Visual odometry - part I: The first 30 years and fundamentals. Robotics \& Automation Magazine, IEEE 18(4), pp. 80-92.

Scaramuzza, D. and Fraundorfer, F., 2012. Visual odometry - part II: Matching, robustness, optimization, and applications. Robotics \& Automation Magazine, IEEE 19(2), pp. 78-90.

Sohnlein, G., Rush, S. and Thompson, L., 2011. Using manned submersibles to create 3D sonar scans of shipwrecks. In: Proceedings of IEEE OCEANS conference, Santander, Spain, pp. 110 .

Tarel, J., Charbonnier, P. and Ieng, S., 2007. Simultaneous robust fitting of multiple curves. In: International Conference on Computer Vision Theory and Applications (VISAPP), Barcelone, Spain, pp. 175-182.

Tarel, J., Ieng, S. and Charbonnier, P., 2008. A constrainedoptimization based half-quadratic algorithm for robustly fitting sets of linearly parametrized curves. Advances in Data Analysis and Classification 2(3), pp. 227-239.

Tomasi, C. and Manduchi, R., 1998. Bilateral filtering for gray and color images. In: ICCV, pp. 839-846. 